\title{
Risk Assessment and Refined Management of Blood Collection and Supply during the COVID-19 Epidemic
}

\author{
Ying Li, Shuchao Zhang, Shaoqiang Zhang, Haiyan Wang* \\ Department of Blood Transfusion, The Affiliated Hospital of Qingdao University, Qingdao, China \\ Email: *why_phd@163.com
}

How to cite this paper: Li, Y., Zhang, S.C., Zhang, S.Q. and Wang, H.Y. (2021) Risk Assessment and Refined Management of Blood Collection and Supply during the COVID-19 Epidemic. Open Journal of Nursing, 11, 668-675.

https://doi.org/10.4236/ojn.2021.118056

Received: July 27, 2021

Accepted: August 17, 2021

Published: August 20, 2021

Copyright $\odot 2021$ by author(s) and Scientific Research Publishing Inc. This work is licensed under the Creative Commons Attribution International License (CC BY 4.0).

http://creativecommons.org/licenses/by/4.0/

\begin{abstract}
Since the end of 2019, severe acute respiratory syndrome coronavirus 2 (SARS$\mathrm{CoV}-2$ ) has continued to spread worldwide and has become a major global public health threat. SARS-CoV-2 has the characteristics of a long incubation period and asymptomatic infection, which are undoubtedly major challenges to blood transfusion safety. Although no research has suggested that there is a risk of SARS-CoV-2 transmission through blood transfusion, the safe use of clinical blood during the epidemic is a serious problem faced by blood collection and supply institutions. Herein, we elaborate on the management of blood collection and supply during the coronavirus disease 2019 (COVID-19) pandemic from aspects such as blood inventory management, clinical blood use, and reducing the risk of transmission of SARS-CoV-2 via blood transfusion. Blood service departments should adopt flexible policies to ensure that blood collection networks can meet clinical needs, while at the same time protecting staff and blood donors, maintaining blood safety, and reducing blood risks during the epidemic.
\end{abstract}

\section{Keywords}

COVID-19, Blood Management, Blood Transfusion Services, Clinical Blood Safety

\section{Introduction}

The global outbreak of the coronavirus disease 2019 (COVID-19) is an immense challenge to public health [1]. The severe acute respiratory syndrome coronavirus 2 (SARS-CoV-2) is mainly transmitted through respiratory tract fluids and physical contact, although there is also a possibility of aerosol transmission [2]. SARS-CoV-2 is the third coronavirus reported to cause severe pneumonia in 
humans after the severe acute respiratory syndrome coronavirus (SARS-CoV) and Middle East respiratory syndrome coronavirus (MERS-CoV) [3] [4]. According to the current epidemiological investigations, SARS-CoV-2 has a longer incubation period and more asymptomatic infections than the other two coronaviruses; the mean incubation period is 6.4 days and ranges from 2.1 to 11.1 days [5]. If volunteers donate blood during the incubation period, there is a risk of viral transmission to vulnerable patients, and this is undoubtedly a major challenge to current blood safety. One study reported that between $4 \%$ and $11 \%$ of all patients with COVID-19 (i.e., $>30,000$ patients) required intensive care unit (ICU) admission [6], with the most severely ill patients transferred to the ICU due to acute respiratory distress syndrome (ARDS) [7] [8]. Blood transfusion is an important auxiliary treatment method in the treatment of critically ill patients [9] [10].

Blood has been in short supply during the COVID-19 epidemic. On the one hand, it is necessary to increase blood resources, but on the other hand, we need to ensure the safe supply of blood. Therefore, there are major challenges in current blood collection and supply. Some recommendations for blood collection and supply management should be carefully discussed, including optimizing blood inventory and clinical blood management in some ways and reducing the risk of SARS-CoV-2 transmission through blood transfusion to ensure clinical blood safety.

\section{Blood Inventory Management under the COVID-19 Epidemic}

In the face of a sudden epidemic, it is almost impossible to recruit a sufficient number of voluntary blood donors and collect enough blood over a month or even a few months. We are now facing important challenges in the management of blood inventories and the smooth running of clinical settings [11] [12] [13]. The blood transfusion departments of general hospitals should actively take measures to ensure emergency blood use by striving to alleviate the conflict between supply and demand through inventory dynamics and expiration early warning strategies, inventory advance, blood adjustment, and by strengthening communication between the blood station and clinic. These measures can maximize the effectiveness of blood resources, so emergency clinical requirements for blood can be met and more patients saved [14].

\subsection{Establishing an Early Warning Mechanism for Blood Inventories}

Blood bank staff should strictly adhere to the guidelines for blood storage, verification and registration, and the use of blood inventory early warning systems. On the basis of blood type, blood is stored in different specialized equipment and clearly marked for identification. Regular blood stock-checking is essential to ensure that the physical items are consistent with the inventory information and that the inventory level meets the 7-day usage rule. If inventory levels are 
abnormal, graded alerts of different colors will appear on a digital database, and the blood bank manager can set the early warning levels for the blood validity period. To coordinate supplies, various hospitals can establish networks, dynamic monitoring systems, and mutual deployment programs. The blood transfusion information system provides early warnings that blood is due to expire within one week and releases those blood products to reduce wastage. To ensure that there is an adequate emergency blood supply, the blood transfusion department should continuously issue blood inventory warnings on the hospital intranet in conjunction with the medical office to ensure that patients with acute and critical illness have the blood they require.

\subsection{Establishing a Blood Inventory Management Mechanism}

It is highly beneficial if blood transfusion doctors can order and retrieve blood on the same day. In cases of pregnant women and emergency and critically ill patients, clinicians should order blood urgently and retrieve it in a timely manner, and the inventory should be updated. There should be regular updates to the blood transfusion information system, the implementation of information and blood supply sharing among various hospitals, and the real-time identification of blood close to the expiration date. Because we need to ensure blood safety, the blood transfusion departments of several hospitals within the jurisdiction may exchange blood after reporting to the blood center which records the exchange. We should aim to establish real-time dynamic adjustments in inventories among hospitals and blood centers; establish a joint mechanism for emergency blood use in an epidemic situation; and keep excellent records of the time, variety, and quantity of blood allocation in blood centers.

\subsection{Paying Attention to the Timing of Blood Distribution}

After receiving a blood transfusion application, the blood transfusion department should supply blood in the order of critically ill, emergency, and routinely stable patients, and elective surgery and chronic anemia patients. Such strategic blood distribution can reduce the peak blood consumption period, avoid medical accidents owing to insufficient blood sources, avoid the queuing and gathering of blood collection personnel, and reduce the probability of cross-infection. At the same time, we should thoroughly publicize the need for blood donations, mobilize blood donation banks, and promote blood fee reductions and exemptions [15].

\section{Blood Transfusion Services during the Epidemic}

In the face of severe shortages of blood stocks and the inability to recruit blood donors normally during a severe epidemic, guaranteeing blood supplies for emergency operations and for patients with acute hemorrhaging is an urgent problem for the blood transfusion department [16]. To ensure the normal progress of clinical work, the following suggestions are made. 1) For patients un- 
dergoing elective surgery, blood transfusion physicians should conduct a comprehensive assessment of the patient's general condition, tolerable blood loss, and estimated blood volume, and the inventory of the corresponding blood type, and provide appropriate blood preparation plans and admission recommendations. 2) For patients undergoing elective surgery, hospitals should consider carrying out autologous blood storage work to minimize the use of allogeneic blood [17]. 3) Blood transfusion doctors should strictly implement the consultation system and use blood in strict accordance with the norms of blood transfusion indications. 4) Blood transfusion doctors and clinicians need to jointly formulate individualized blood-use plans to avoid clinically empirical blood use and reduce wastage of blood resources.

\section{Reducing the Risk of SARS-CoV-2 Transmission through Blood Transfusion to Ensure Clinical Blood Safety}

Blood transfusion is an important part of clinical treatment. During an epidemic, it is necessary to ensure blood collection and blood safety to ensure the smooth progression of patient treatment [18]. On January 31, 2020, the American Association of Blood Banks (AABB) issued recommendations on the impact of SARSCoV-2 on blood safety. So far, there has been no report of SARS-CoV-2 being transmitted through blood or other human bodily fluids or tissue, and the same is true for the other two coronaviruses (SARS-CoV and MERS-CoV) that have emerged in the past two decades [19] [20] [21]. Because there are currently no relevant data or blood transfusion infection cases that suggest that SARS-CoV-2 transmission is a risk of transfusion, the Food and Drug Administration (FDA) and the Center for Disease Control and Prevention have not yet published recommendations for blood collection and supply institutions. However, in response to public concerns about blood supply and safety, blood collection and supply institutions can take voluntary preventive measures.

\subsection{Excluding High-Risk Blood Donors}

As a preventive measure, blood collection and supply institutions could establish health consultation and notification systems for blood donors during the epidemic in addition to regular blood donation consultation. To determine whether a volunteer is eligible for blood donation, the staff need to routinely disinfect the donors' hands and skin around the area used to draw blood, check their body temperature, and arrange for the responsible physician to conduct detailed inquiries into their history of recent travel and residence in the affected area, contact with infected persons, and whether they have related symptoms, such as fever and fatigue. On the basis of the existing information, the FDA recommends that blood donors with a history of infection or contact with infected people should defer blood donation for at least 28 days. Donors who are diagnosed after the blood donation must promptly report to the blood collection and supply unit and clinic. The donor should take immediate isolation measures, and 
the donated blood should be discarded. In addition, the risk of blood-borne epidemics should be monitored in real-time, and new key recommendations should be implemented in a timely manner to ensure the safe supply of blood.

\subsection{Self-Protection of Staff}

Asymptomatic virus carriers and patients with pre-acute infections may become transmission vectors [22]. During epidemic prevention and control periods, the staff of blood collection and supply units face potential risks during routine blood collection and laboratory testing [23], and they should screen blood donors strictly while adopting first-level biological safety protection measures (medical surgical masks, medical protective caps, overalls, gloves), wear goggles when collecting blood, and carry out strict hand hygiene practice and disinfection of the blood collection environment. For those wards dealing with apheresis treatment, first-level bio-safety protection is adopted in general wards, and secondlevel bio-safety protection is required in isolation wards and infected wards. General specimen-testing laboratory personnel should adopt first-level biosafety protection, and second-level biosafety protection is required when handling infected specimens. In addition to respiratory transmission, aerosol transmission may also be a mode of SARS-CoV-2 transmission [24]. Processing the blood samples of COVID-19 patients is likely to produce large quantities of aerosols, especially when a centrifuge is running at high speed, posing a potential threat to laboratory personnel. Therefore, it is recommended that a decap centrifuge is used when testing confirmed or suspected specimens, and it is good practice to use a biological safety cabinet for specimen manipulation and processing. The air is drawn out of biological safety cabinets, so that the cabinet is under negative pressure [25]. Air enters the cabinet after passing through an air filter, which can protect the specimens in the operation area from pollution, and operators from exposure to aerosols when handling infectious specimens.

\subsection{Reducing Pathogens in the Blood}

The AABB's recommendations on the impact of SARS-CoV-2 on blood safety state that if there is evidence that SARS-CoV-2 has a risk of transmission through blood transfusion, reducing pathogens may be another option to ensure blood safety. Pathogen reduction technology (PRT) can be used when working with platelets and plasma but not red blood cells. PRT has been proven to effectively resist SARS-CoV when applied to platelets and plasma [26]. Riboflavin-based and ultraviolet light-based photochemical treatments can effectively reduce the titer of MERS-CoV virus in human plasma to below the detection limit; therefore, this treatment method can reduce the risk of MERS-CoV virus transmission through blood transfusion [27]. Whether PRT, riboflavin, and ultraviolet photochemical methods are effective for controlling SARS-CoV-2 remains to be further studied. No detectable antibodies are formed in the patient's body during the acute phase of viral infection, thus serological testing is not effective for 
SARS-CoV-2 infection screening. Nucleic acid testing (NAT) for SARS-CoV-2 can minimize the risk of transmission through blood transfusion and guarantee the safety of the blood from the point of source. However, there have been few studies on the applicability and effectiveness of NAT as a screening test for asymptomatic blood donors, and the cost of NAT is very high. For example, more than 4,000,000 donation NATs were conducted to check for ZIKA virus in certain areas of the USA, at a cost of 5.3 million US dollars, and eight cases in total were reactive [28]. At present, many blood stations in China, including those in Jinan City, Yuxi City, Xiangyang City, and Chenzhou City, are detecting SARS-CoV-2 nucleic acids in the blood of donors. Until now, all NAT results from blood collection and supply institutions have been negative, and no positive cases have emerged. It is still unknown if SARS-CoV-2 or similar coronaviruses can be transmitted by blood transfusion, and the screening of blood donors is currently very strict, which has further reduced the theoretical risk of virus transmission through blood transfusion. Therefore, it remains to be explored if introducing NAT or PRT for blood screening of blood donors is necessary.

\section{Conclusion}

At the time of writing, SARS-CoV-2 continues to spread globally. After active classification studies, scientists have a preliminary understanding of SARS-CoV-2, but its epidemiology needs further in-depth exploration. In the face of sudden epidemics, blood service departments should adopt flexible policies and actively prepare emergency plans to ensure that blood collection networks can meet clinical needs, while at the same time protecting staff and blood donors, maintaining blood safety, and reducing blood risks during the epidemic. Given that the current impact of SARS-CoV-2 on blood safety is theoretical, blood collection and supply institutions need to periodically reassess donor risks and preventive exclusion strategies as the epidemic progresses. In the case of widespread epidemics, shortage of blood products due to the reduction of donors in each region is a huge challenge. Therefore, blood collection and supply institutions should consider the risks of blood supply shortages as soon as possible.

\section{Conflicts of Interest}

The authors declare no conflicts of interest regarding the publication of this paper.

\section{References}

[1] Jin, Y.H., Cai, L., Cheng, Z.S., Cheng, H., Deng, T., Fan, Y.P., et al. (2020) A Rapid Advice Guideline for the Diagnosis and Treatment of 2019 Novel Coronavirus (2019nCoV) Infected Pneumonia(Standard Version). Military Medical Research, 7, Article No. 4. https://doi.org/10.1186/s40779-020-0233-6

[2] Jayaweera, M., Perera, H., Gunawardana, B. and Manatunge, J. (2020) Transmission of COVID-19 Virus by Droplets and Aerosols: A Critical Review on the Unresolved Dichotomy. Environmental Research, 188, Article ID: 109819. 
https://doi.org/10.1016/j.envres.2020.109819

[3] Petrosillo, N., Viceconte, G., Ergonul, O., Ippolito, G. and Petersen, E. (2020) COVID-19, SARS and MERS: Are They Closely Related? Clinical Microbiology and Infection, 26, 729-734. https://doi.org/10.1016/j.cmi.2020.03.026

[4] Harrison, A.G., Lin, T. and Wang, P. (2020) Mechanisms of SARS-CoV-2 Transmission and Pathogenesis. Trends in Immunology, 41, 1100-1115. https://doi.org/10.1016/j.it.2020.10.004

[5] Backer, J.A., Klinkenberg, D. and Wallinga, J. (2020) Incubation Period of 2019 Novel Coronavirus (2019-nCoV) Infections among Travellers from Wuhan, China, 20-28 January 2020. Eurosurveillance, 25, Article ID: 2000062. https://doi.org/10.2807/1560-7917.ES.2020.25.5.2000062

[6] Lew, H.L., Oh-Park, M. and Cifu, D.X. (2020) The War on COVID-19 Pandemic: Role of Rehabilitation Professionals and Hospitals. American Journal of Physical Medicine \& Rehabilitation, 99, 571-572. https://doi.org/10.1097/PHM.0000000000001460

[7] Teymoori-Rad, M., Samadizadeh, S., Tabarraei, A., Moradi, A., Shahbaz, M.B. and Tahamtan, A. (2020) Ten Challenging Questions about SARS-CoV-2 and COVID19. Expert Review of Respiratory Medicine, 14, 881-888. https://doi.org/10.1080/17476348.2020.1782197

[8] Yu, Y., Xu, D., Fu, S., Zhang, J., Yang, X., Xu, L., et al. (2020) Patients with COVID-19 in 19 ICUs in Wuhan, China: A Cross-Sectional Study. Critical Care 24, Article No. 219. https://doi.org/10.1186/s13054-020-02939-X

[9] Vincent, J.L., Baron, J.F., Reinhart, K., Gattinoni, L., Thijs, L., Webb, A., et al. (2002) Anemia and Blood Transfusion in Critically Ill Patients. JAMA, 288, 1499-507. https://doi.org/10.1001/jama.288.12.1499

[10] Spinella, P.C., Tucci, M., Fergusson, D.A., Lacroix, J., Hébert, P.C., Leteurtre, S., et al. (2019) Effect of Fresh vs Standard-Issue Red Blood Cell Transfusions on Multiple Organ Dysfunction Syndrome in Critically Ill Pediatric Patients: A Randomized Clinical Trial. JAMA, 322, 2179-2190.

https://doi.org/10.1001/jama.2019.17478

[11] Stanworth, S.J., New, H.V., Apelseth, T.O., Brunskill, S., Cardigan, R., Doree, C., et al. (2020) Effects of the COVID-19 Pandemic on Supply and Use of Blood for Transfusion. The Lancet Haematology, 7, e756-e764. https://doi.org/10.1016/S2352-3026(20)30186-1

[12] Ngo, A., Masel, D., Cahill, C., Blumberg, N. and Refaai, M.A. (2020) Blood Banking and Transfusion Medicine Challenges during the COVID-19 Pandemic. Clinics in Laboratory Medicine, 40, 587-601. https://doi.org/10.1016/j.cll.2020.08.013

[13] Raturi, M. and Kusum, A. (2020) The Blood Supply Management Amid the COVID19 Outbreak. Transfusion Clinique et Biologique, 27, 147-151. https://doi.org/10.1016/j.tracli.2020.04.002

[14] Gehrie, E.A., Frank, S.M. and Goobie, S.M. (2020) Balancing Supply and Demand for Blood during the COVID-19 Pandemic. Anesthesiology, 133, 16-18. https://doi.org/10.1097/ALN.0000000000003341

[15] Sahu, K.K., Raturi, M., Siddiqui, A.D. and Cerny, J. (2020) "Because Every Drop Counts": Blood Donation during the COVID-19 Pandemic. Transfusion Clinique et Biologique, 27, 105-108. https://doi.org/10.1016/j.tracli.2020.06.009

[16] Baron, D.M., Franchini, M., Goobie, S.M., Javidroozi, M., Klein, A.A., Lasocki, S., et al. (2020) Patient Blood Management during the COVID-19 Pandemic: A Narrative Review. Anaesthesia, 75, 1105-1113. https://doi.org/10.1111/anae.15095 
[17] Frank, S.M., Sikorski, R.A., Konig, G., Tsilimigras, D.I., Hartmann, J., Popovsky, M.A., et al. (2020) Clinical Utility of Autologous Salvaged Blood: A Review. Journal of Gastrointestinal Surgery, 24, 464-472.

https://doi.org/10.1007/s11605-019-04374-y

[18] Dodd, R.Y. and Stramer, S.L. (2020) COVID-19 and Blood Safety: Help with a Dilemma. Transfusion Medicine Reviews, 34, 73-74. https://doi.org/10.1016/j.tmrv.2020.02.004

[19] Ng, E.K., Hui, D.S., Chan, K.C., Hung, E.C.W., Chiu, R.W.K., Lee, N., et al. (2003) Quantitative Analysis and Prognostic Implication of SARS Coronavirus RNA in the Plasma and Serum of Patients with Severe Acute Respiratory Syndrome. Clinical Chemistry, 49, 1976-1980. https://doi.org/10.1373/clinchem.2003.024125

[20] Chang, L., Yan, Y. and Wang, L. (2020) Coronavirus Disease 2019: Coronaviruses and Blood Safety. Transfusion Medicine Reviews, 34, 75-80.

https://doi.org/10.1016/j.tmrv.2020.02.003

[21] Corman, V.M., Rabenau, H.F., Adams, O., Oberle, D., Funk, M.B., Keller-Stanislawski, B., et al. (2020) SARS-CoV-2 Asymptomatic and Symptomatic Patients and Risk for Transfusion Transmission. Transfusion, 60, 1119-1122.

https://doi.org/10.1111/trf.15841

[22] Gao, Z., Xu, Y., Sun, C., Wang, X., Guo, Y., Qiu, S., et al. (2021) A Systematic Review of Asymptomatic Infections with COVID-19. Journal of Microbiology, Immunology and Infection, 54, 12-16. https://doi.org/10.1016/j.jmii.2020.05.001

[23] Frater, J.L., Zini, G., d'Onofrio, G. and Rogers, H.J. (2020) COVID-19 and the Clinical Hematology Laboratory. International Journal of Laboratory Hematology, 42, 11-18. https://doi.org/10.1111/ijlh.13229

[24] Sommerstein, R., Fux, C.A., Vuichard-Gysin, D., Abbas, M., Marschall, J., Balmelli, C., et al. (2020) Risk of SARS-CoV-2 Transmission by Aerosols, the Rational Use of Masks, and Protection of Healthcare Workers from COVID-19. Antimicrobial Resistance \& Infection Control, 9, Article No. 100. https://doi.org/10.1186/s13756-020-00763-0

[25] Dondelinger, R. (2013) Biological Safety Cabinets. Biomedical Instrumentation \& Technology, 47, 333-338. https://doi.org/10.2345/0899-8205-47.4.333

[26] Eickmann, M., Gravemann, U., Handke, W., Tolksdorf, F., Reichenberg, S., Müller, T.H., et al. (2020) Inactivation of Three Emerging Viruses-Severe Acute Respiratory Syndrome Coronavirus, Crimean-Congo Haemorrhagic Fever Virus and Nipah Virus-In Platelet Concentrates by Ultraviolet C Light and in Plasma by Methylene Blue Plus Visible Light. Vox Sanguinis, 115, 146-151.

https://doi.org/10.1111/vox.12888

[27] Keil, S.D., Bowen, R. and Marschner, S. (2016) Inactivation of Middle East Respiratory Syndrome Coronavirus (MERS-CoV) in Plasma Products Using a RiboflavinBased and Ultraviolet Light-Based Photochemical Treatment. Transfusion, 56, 2948 2952. https://doi.org/10.1111/trf.13860

[28] Saá, P., Proctor, M., Foster, G., Krysztof, D., Winton, C., Linnen, J.M., et al. (2018) Investigational Testing for Zika Virus among U.S. Blood Donors. New England Journal of Medicine, 378, 1778-1788. https://doi.org/10.1056/NEJMoa1714977 